Direct Methods for Solving Macromolecular Structures

Ed. by S. Fortier

Kluwer Academic Publishes, The Netherlands, 1998, pp. 297-305.

\title{
DIRECT METHODS OF SOLVING PHASE AMBIGUITIES IN MACRO- MOLECULAR STRUCTURES
}

\author{
FAN HAI-FU \\ Institute of Physics, Chinese Academy of Sciences \\ Beijing 100080, P. R. China
}

\section{Notation}

h the reciprocal-lattice vector, which corresponds to the diffraction index $h k l$

$\mathbf{F}_{\mathbf{h}}$ the structure factor at the reciprocal-lattice node defined by $\mathbf{h}$

$F_{\mathbf{h}}$ the magnitude of $\mathbf{F}_{\mathbf{h}}$

$\varphi_{\mathrm{h}}$ the phase of $\mathbf{F}_{\mathbf{h}}$

$\mathbf{E}_{\mathbf{h}}$ the normalised structure factor corresponding to $\mathbf{F}_{\mathbf{h}}$

$E_{\mathbf{h}}$ the magnitude of $\mathbf{E}_{\mathbf{h}}$

$\mathbf{F}_{\mathbf{h}}^{\mathrm{o}}$ the structure-factor contribution from the non-anomalous scatterers

$\mathbf{F}_{\mathbf{h}, A}$ the structure-factor contribution from the real-part scattering of the anomalous scatterers, i.e.

$$
\mathbf{F}_{\mathbf{h}, A}^{\prime}=\sum_{A=1}^{N_{A}}\left(f_{A}+\Delta f^{\prime}{ }_{A}\right) \exp \left(i 2 \pi \mathbf{h} \cdot \mathbf{r}_{A}\right)
$$

$\mathbf{F}{ }_{\mathbf{h}, A} \quad$ the structure-factor contribution from the imaginary-part scattering of the anomalous scatterers, i.e.

$$
\mathbf{F}_{\mathbf{h}, A}=\sum_{A=1}^{N_{A}} i \Delta f^{\prime \prime} \exp \left(i 2 \pi \mathbf{h} \cdot \mathbf{r}_{A}\right)
$$

$\sigma_{n}=\Sigma_{j}\left(Z_{j}\right)^{n}, Z_{j}$ is the atomic number of the $j^{\text {th }}$ atom in the unit cell, $n$ is an integer equal to 2 or 3

$\sigma_{u}=\Sigma_{u}\left(Z_{u}\right)^{2} / \sigma_{2}, Z_{u}$ is the atomic number of the $u^{\text {th }}$ atom, which belongs to the unknown part of the structure

\section{Subscripts:}

$A$ the anomalous scattering atoms

$R$ the replacing atoms of an isomorphous pair

$N$ the atoms in the native protein

$D$ the atoms in the heavy-atom derivative of the native protein

$p$ the atoms of the partial structure with known positions in the unit cell

$u$ the atoms of the unknown part in the unit cell

\section{Introduction}


Solving a macromolecular structure by using single isomorphous replacement (SIR) or one wavelength anomalous scattering (OAS) data is important when it is difficult to prepare multiple isomorphous derivatives and to collect multiwavelength anomalous diffraction data. However the phase ambiguity intrinsic in SIR or OAS data obstructs the use of them. Various kinds of direct methods have been proposed to solve the problem. [1 - 13]. Some of them have been successfully tested with experimental protein-diffraction data [14 - 18]. In this paper the methods of Fan et al. [9, 14] will be discussed in detail.

\section{The phase ambiguity in SIR and OAS cases}

In the case of SIR for a given reciprocal-lattice vector $\mathbf{h}$, we have

$$
\mathbf{F}_{\mathbf{h}, D}-\mathbf{F}_{\mathbf{h}, N}=\mathbf{F}_{\mathbf{h}, R}
$$

From experiment we know the magnitudes of $\mathbf{F}_{\mathbf{h}, \boldsymbol{D}}$ and $\mathbf{F}_{\mathbf{h}, N}$. Accordingly, we can find the positions of the replacing atoms and calculate the vector $\mathbf{F}_{\mathbf{h}, R}$. Thus we have two different ways to draw the triangle of (1) leading to an enantiomorphous phase doublet for $\mathbf{F}_{\mathbf{h}, \boldsymbol{D}}$ or for $\mathbf{F}_{\mathbf{h}, N}$, as shown on the left part of figure 1. In the OAS case, we have

$$
\mathbf{F}_{\mathbf{h}}^{+}-\mathbf{F}_{\mathbf{h}}^{-^{*}}=2 \mathbf{F}_{\mathbf{h}, A}^{\prime \prime}
$$

The magnitudes of $\mathbf{F}^{+}{ }_{\mathbf{h}}$ and $\mathbf{F}^{-*_{\mathbf{h}}}$ can be obtained from experiment, while $\mathbf{F}{ }^{\prime}{ }_{\mathbf{h}, \mathrm{A}}$ can be derived. Hence we have also two ways to draw the triangle of (2) leading to an enantiomorphous phase doublet for $\mathbf{F}_{\mathbf{h}}$, as shown on the right part of figure 1.

The phase doublet in both SIR and OAS cases can be expressed as

$$
\varphi_{\mathbf{h}}=\varphi_{\mathbf{h}} \pm\left|\Delta \varphi_{\mathbf{h}}\right|
$$

In the SIR case

$$
\begin{aligned}
& \varphi_{\mathrm{h}}^{\prime}=\varphi_{\mathrm{h}, \mathrm{R}} \\
& \Delta \varphi_{\mathbf{h}}= \pm \cos ^{-1}\left(F_{\mathbf{h}, \boldsymbol{D}}^{2}-F_{\mathbf{h}, N}^{2}-F_{\mathbf{h}, \boldsymbol{R}}^{2} / 2 F_{\mathbf{h}, \boldsymbol{N}} F_{\mathbf{h}, \boldsymbol{R}}\right)
\end{aligned}
$$

if $\varphi_{\mathbf{h}}$ denotes the phase of a reflection from the native protein;

$$
\Delta \varphi_{\mathbf{h}}= \pm \cos ^{-1}\left(F_{\mathbf{h}, \boldsymbol{D}}^{2}+F_{\mathbf{h}, \boldsymbol{R}}^{2}-F_{\mathbf{h}, \boldsymbol{R}}^{2} / 2 F_{\mathbf{h}, \boldsymbol{D}} F_{\mathbf{h}, \boldsymbol{R}}\right)
$$

if $\varphi_{\mathbf{h}}$ denotes the phase of a reflection from the derivative. 

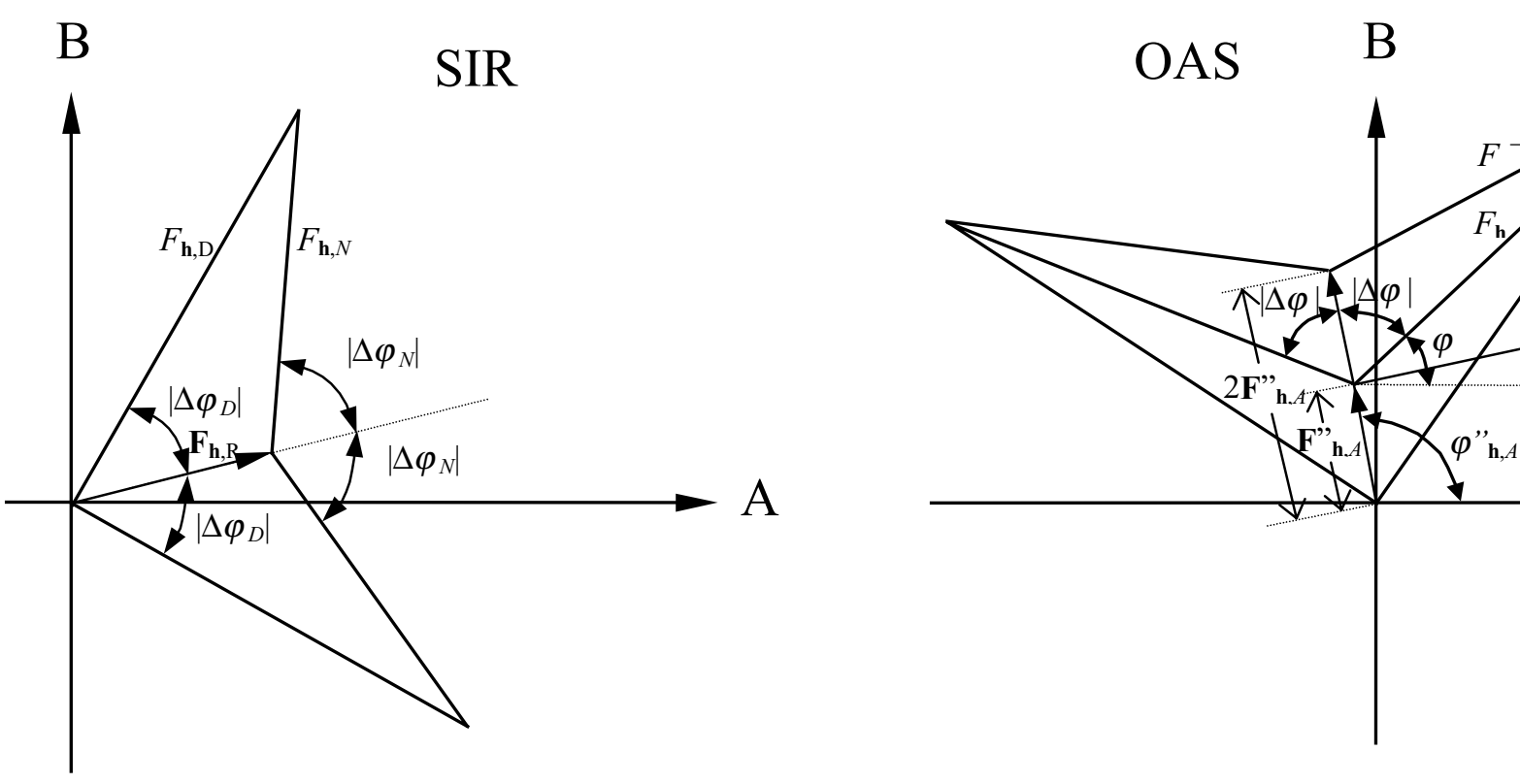

Figure 1. Phase ambiguity (doublets) arising in the single isomorphous replacement (SIR) and one-wavelength anomalous scattering (OAS) cases 
In the OAS case

$$
\begin{gathered}
\varphi_{\mathbf{h}}^{\prime}=\varphi_{\mathbf{h}, A}^{\prime \prime} \\
\Delta \varphi_{\mathbf{h}}= \pm \cos ^{-1}\left(F^{+}{ }_{\mathbf{h}}+F^{-}{ }_{\mathbf{h}} / 2 F_{\mathbf{h}, A}\right)
\end{gathered}
$$

$\varphi_{\mathrm{h}}$ now denotes the phase of

$$
\mathbf{F}_{\mathbf{h}}=\left(\mathbf{F}^{+}{ }_{\mathbf{h}}+\mathbf{F}^{-*}{ }_{\mathbf{h}}\right) / 2
$$

where $\mathbf{F}^{-*}{ }_{\mathbf{h}}$ is the conjugate of $\mathbf{F}^{-}{ }_{\mathbf{h}}$.

To summarise, the problem of splitting the above enantiomorphous phase doublets can be converted into that of finding the sign of $\Delta \varphi_{\mathrm{h}}$ given the known values of $\varphi_{\mathrm{h}}{ }_{\mathrm{h}}$ and $\left|\Delta \varphi_{\mathbf{h}}\right|$. Alternatively, defining

and

$$
A^{\prime}{ }_{\mathbf{h}}=F_{\mathbf{h}} \cos \Delta \varphi_{\mathbf{h}}
$$

$$
B_{\mathbf{h}}=F_{\mathbf{h}} \sin \Delta \varphi_{\mathbf{h}}
$$

the problem becomes that of finding the sign of $B^{\prime}{ }_{\mathbf{h}}$ from the known values of $A_{\mathbf{h}}{ }_{\mathbf{h}}$ and $\left|B^{\prime}{ }_{\mathbf{h}}\right|$.

\section{The phase difference relations}

We start from a modified Sayre's equation [2]:

$$
\mathbf{F}_{\mathbf{h}}=\left(\theta_{\mathbf{h}, u} / V\right) \sum_{\mathbf{h}}, \mathbf{F}_{\mathbf{h}}, \mathbf{F}_{\mathbf{h}-\mathbf{h}},-\sum_{p}\left[\left(\theta_{\mathbf{h}, u} / \theta_{\mathbf{h}, p}\right)-1\right] \mathbf{F}_{\mathbf{h}, p},
$$

where $\theta$ is an atomic form factor, the subscript $u$ denotes the unknown part of the structure consisting of light atoms, while $p$ denotes the partial structure of known heavy atoms. Replacing $\mathbf{F}_{\mathbf{h}}$ by $F_{\mathbf{h}} \exp \left(i \varphi_{\mathbf{h}}\right)$ and replacing $\varphi_{\mathbf{h}}$ by $\varphi_{\mathbf{h}}{ }_{\mathbf{h}}+\Delta \varphi_{\mathbf{h}}$, we obtain [19]:

$$
\begin{aligned}
& F_{\mathbf{h}} \exp \left(i \Delta \varphi_{\mathbf{h}}\right)=\left(\theta_{\mathbf{h}, u} / V\right) \sum_{\mathbf{h}}, F_{\mathbf{h}^{\prime}} F_{\mathbf{h}-\mathbf{h}^{\prime}} \\
& \quad \times \exp \left[i \left(-\varphi_{\mathbf{h}}^{\prime}+\varphi_{\mathbf{h}^{\prime}}^{\prime}+\varphi_{\mathbf{h}-\mathbf{h}^{\prime}}^{\prime}+\Delta \varphi_{\mathbf{h}^{\prime}}+\Delta \varphi_{\left.\left.\mathbf{h}-\mathbf{h}^{\prime}\right)\right]}\right.\right. \\
& \quad-\sum_{p}\left[\left(\theta_{\mathbf{h}, u} / \theta_{\mathbf{h}, p}\right)-1\right] F_{\mathbf{h}, p} \exp \left[i\left(\varphi_{\mathbf{h}, p}-\varphi_{\mathbf{h}}^{\prime}\right)\right] .
\end{aligned}
$$

Taking the imaginary part of (13), it follows 


$$
\begin{gathered}
F_{\mathbf{h}} \sin \Delta \varphi_{\mathbf{h}}=\left(\theta_{\mathbf{h}, u} / V\right) \sum_{\mathbf{h}}, F_{\mathbf{h}}, F_{\mathbf{h}-\mathbf{h}}, \sin \left(\Phi^{\prime}{ }_{3}+\Delta \varphi_{\mathbf{h}}, \Delta \varphi_{\mathbf{h}-\mathbf{h}}\right) \\
-\sum_{p}\left[\left(\theta_{\mathbf{h}, u} / \theta_{\mathbf{h}, p}\right)-1\right] F_{\mathbf{h}, p} \sin \left(\varphi_{\mathbf{h}, p}-\varphi_{\mathbf{h}}\right)
\end{gathered}
$$

or equivalently

$$
\begin{gathered}
B_{\mathbf{h}}^{\prime}=\left(\theta_{\mathbf{h}, u} / V\right)\left[\sum_{\mathbf{h}},\left(A^{\prime}{ }_{\mathbf{h}}, A^{\prime}{ }_{\mathbf{h}-\mathbf{h}}{ }^{\prime}-B^{\prime}{ }_{\mathbf{h}}, B^{\prime}{ }_{\mathbf{h}-\mathbf{h}}\right) \sin \Phi_{3}^{\prime}\right. \\
\left.+2 \sum_{\mathbf{h}}, A^{\prime}{ }_{\mathbf{h}}{ }^{\prime} B^{\prime}{ }_{\mathbf{h}-\mathbf{h}}, \cos \Phi^{\prime}{ }_{3}\right] \\
-\sum_{p}\left[\left(\theta_{\mathbf{h}, u} / \theta_{\mathbf{h}, p}\right)-1\right] F_{\mathbf{h}, p} \sin \left(\varphi_{\mathbf{h}, p}-\varphi_{\mathbf{h}}\right)
\end{gathered}
$$

where

$$
\Phi_{3}^{\prime}=-\varphi_{\mathbf{h}}^{\prime}+\varphi_{\mathbf{h}^{\prime}}^{\prime}+\varphi_{\mathbf{h}^{-\mathbf{h}^{\prime}}}^{\prime}
$$

Equation (14) is the 'phase-difference relation', while (15) may be regarded as a 'modified component relation'. In the case of SIR with a centrosymmetric heavy-atom arrangement (15) reduces to one of the original component relations [3]:

$$
B_{\mathbf{h}}=\left(2 \theta_{\mathbf{h}, u} / V\right) \sum_{\mathbf{h}}, A_{\mathbf{h}}, B_{\mathbf{h}-\mathbf{h}},-\sum_{p}\left[\left(\theta_{\mathbf{h}, u} / \theta_{\mathbf{h}, p}\right)-1\right] B_{\mathbf{h}, p},
$$

while in the case of OAS with a centrosymmetric heavy-atom arrangement, (15) reduces to the other original component relation:

$$
\begin{aligned}
A_{\mathbf{h}}= & \left(\theta_{\mathbf{h}, u} / V\right) \sum_{\mathbf{h}},\left(A_{\mathbf{h}}, A_{\mathbf{h}-\mathbf{h}}, B_{\mathbf{h}}, B_{\mathbf{h}-\mathbf{h}}\right) \\
& -\sum_{p}\left[\left(\theta_{\mathbf{h}, u} / \theta_{\mathbf{h}, p}\right)-1\right] A_{\mathbf{h}, p} .
\end{aligned}
$$

Here $A_{\mathbf{h}}$ and $B_{\mathbf{h}}$ are the real and imaginary components of the structure factor, respectively.

With (14) or (15) it is possible to derive the signs of $\Delta \varphi_{\mathbf{h}}$ or $B^{\prime}{ }_{\mathbf{h}}$ by a simple procedure like that of using Sayre's equation [20] for a centrosymmetric structure [21]. A special case, which should be mentioned here, is that a centrosymmetric heavy-atom arrangement in a non-centrosymmetric crystal causes phase ambiguity even for small structures. If this occurs with a set of SIR protein data, we get a 'two-fold' phase ambiguity. The solvent flattening technique $[22,23]$ can not break the SIR phase ambiguity in this case, although it works quite effectively otherwise. On the other hand equation (17) can easily resolve the phase ambiguity under this circumstance, since we know already $A_{\mathbf{h}}, B_{\mathbf{h}, p}$ and the absolute value of $B_{\mathbf{h}}$. The only problem is to find the signs for $B_{\mathbf{h}}$ by making use of equation (17), which relates the real and the imaginary parts of structure factors, $A_{\mathbf{h}}$ and $B_{\mathbf{h}}$. 


\section{A tangent-formula among $\Delta \varphi_{\mathrm{h}}$ and the probability of $\Delta \varphi_{\mathrm{h}}$ to be positive}

According to Cochran [24], for a set of triplets $\varphi_{\mathbf{h}}-\varphi_{\mathbf{h}},-\varphi_{\mathbf{h}-\mathbf{h}^{\prime}}$, the conditional probability distribution of $\varphi_{\mathbf{h}}$ given $E_{\mathbf{h}}, E_{\mathbf{h}^{\prime}}, E_{\mathbf{h}-\mathbf{h}^{\prime}} \cdot \varphi_{\mathbf{h}^{\prime}}$ and $\varphi_{\mathbf{h}-\mathbf{h}^{\prime}}$ is as follows:

$$
P\left(\varphi_{\mathbf{h}}\right)=N \exp \left[\sum_{\mathbf{h}^{\prime}} \kappa_{\mathbf{h}^{\prime}} \cos \left(\varphi_{\mathbf{h}}-\varphi_{\mathbf{h}^{\prime}}-\varphi_{\mathbf{h}-\mathbf{h}^{\prime}}\right)\right],
$$

where $\mathrm{N}$ is a normalizing factor and

$$
\kappa_{\mathbf{h}, \mathbf{h}^{\prime}}=2 \sigma_{3} \sigma_{2}^{-3 / 2} E_{\mathbf{h}} E_{\mathbf{h}^{\prime}} E_{\mathbf{h}-\mathbf{h}^{\prime}}
$$

With equation (16) and the expression $\varphi_{\mathbf{h}}=\varphi^{\prime}{ }_{\mathbf{h}}+\Delta \varphi_{\mathbf{h}}$, the triplet phase becomes

$$
\varphi_{\mathbf{h}}-\varphi_{\mathbf{h}},-\varphi_{\mathbf{h}-\mathbf{h}},=\Delta \varphi_{\mathbf{h}}-\left(\Phi^{\prime}{ }_{3}+\Delta \varphi_{\mathbf{h}},+\Delta \varphi_{\mathbf{h}-\mathbf{h}^{\prime}}\right)
$$

Substitution of (20) into (19) gives

$$
P\left(\Delta \varphi_{\mathbf{h}}\right)=N \exp \left[\sum_{\mathbf{h}^{\prime}} \kappa_{\mathbf{h}, \mathbf{h}^{\prime}} \cos \left[\Delta \varphi_{\mathbf{h}}-\left(\Phi_{3}^{\prime}+\Delta \varphi_{\mathbf{h}^{\prime}}+\Delta \varphi_{\mathbf{h}-\mathbf{h}^{\prime}}\right)\right]\right.
$$

Let

and

$$
\alpha \sin \beta=\sum_{\mathbf{h}}, \kappa_{\mathbf{h}, \mathbf{h}}, \sin \left[\Phi_{3}^{\prime}+\Delta \varphi_{\mathbf{h}},+\Delta \varphi_{\mathbf{h}-\mathbf{h}^{\prime}}\right]
$$

$$
\alpha \cos \beta=\sum_{\mathbf{h}} \kappa_{\mathbf{h}, \mathbf{h}}, \cos \left[\Phi_{3}^{\prime}+\Delta \varphi_{\mathbf{h}},+\Delta \varphi_{\mathbf{h}-\mathbf{h}}\right]
$$

(21) becomes

$$
P\left(\Delta \varphi_{\mathbf{h}}\right)=\left[2 \pi I_{0}(\alpha)\right]^{-1} \exp \left[\alpha \cos \left(\Delta \varphi_{\mathbf{h}}-\beta\right)\right],
$$

where $I_{0}(\alpha)$ is the zero-order modified Bessel function of the first kind with $\alpha$ as argument. Equation (24) is the probability distribution of $\Delta \varphi_{\mathrm{h}}$ based on Cochran's distribution. By maximising (24) we have $\beta=\Delta \varphi_{\mathrm{h}}$ and hence from (22) and (23) it follows

$$
\tan \Delta \varphi_{\mathbf{h}}=\frac{\sum_{\mathbf{h}^{\prime}} \kappa_{\mathbf{h}, \mathbf{h}^{\prime}} \sin \left(\Phi_{3}^{\prime}+\Delta \varphi_{\mathbf{h}^{\prime}}+\Delta \varphi_{\mathbf{h}-\mathbf{h}^{\prime}}\right)}{\sum_{\mathbf{h}^{\prime}} \kappa_{\mathbf{h}, \mathbf{h}^{\prime}} \cos \left(\Phi_{3}^{\prime}+\Delta \varphi_{\mathbf{h}^{\prime}}+\Delta \varphi_{\mathbf{h}-\mathbf{h}^{\prime}}\right)}
$$


This is a tangent formula relating the phase differences.

On the other hand, according to Sim [25], if partial structure information is available, we have

where

$$
P_{\text {Sim }}(\theta)=\left[2 \pi I_{0}(x)\right]^{-1} \exp [x \cos (\theta)],
$$

$$
\mathrm{x}=2 E_{\mathbf{h}} E_{\mathbf{h}, p} / \sigma_{u}, \quad \theta=\varphi_{\mathbf{h}}-\varphi_{\mathbf{h}, p}
$$

With the expression $\varphi_{\mathbf{h}}=\varphi^{\prime}{ }_{\mathrm{h}}+\Delta \varphi_{\mathrm{h}},(26)$ becomes

$$
P_{S i m}\left(\Delta \varphi_{\mathbf{h}}\right)=\left[2 \pi I_{0}(x)\right]^{-1} \exp \left[x \cos \left(\Delta \varphi_{\mathbf{h}}-\delta_{\mathbf{h}}\right)\right],
$$

where

$$
\delta_{\mathbf{h}}=\varphi_{\mathbf{h}, p}-\varphi_{\mathbf{h}}
$$

By multiplying equations (27) and (24), and by introducing the concept of 'best phase relationship' [26], we finally obtain a tangent formula for phase differences $\Delta \varphi_{\mathbf{h}}$, which is based on Cochran's distribution and Sim's distribution and the concept of 'best phases' in protein crystallography:

$$
\tan \Delta \varphi_{\mathbf{h}}=\frac{\sum_{\mathbf{h}^{\prime}} m_{\mathbf{h}^{\prime}} m_{\mathbf{h}-\mathbf{h}^{\prime}} \kappa_{\mathbf{h}, \mathbf{h}^{\prime}} \sin \left(\Phi_{3}^{\prime}+\Delta \varphi_{\mathbf{h}^{\prime} \text { best }}+\Delta \varphi_{\mathbf{h}-\mathbf{h}^{\prime} \text { best }}\right)+x \sin \delta_{\mathbf{h}}}{\sum_{\mathbf{h}^{\prime}} m_{\mathbf{h}^{\prime}} m_{\mathbf{h}-\mathbf{h}^{\prime}} \kappa_{\mathbf{h}, \mathbf{h}^{\prime}} \cos \left(\Phi_{3}^{\prime}+\Delta \varphi_{\mathbf{h}^{\prime} \text { best }}+\Delta \varphi_{\mathbf{h}-\mathbf{h}^{\prime} \text { best }}\right)+x \cos \delta_{\mathbf{h}}}
$$

with the corresponding probability

$$
\begin{aligned}
& P_{+}\left(\Delta \varphi_{\mathbf{h}}\right)=\frac{1}{2}+\frac{1}{2} \tanh \left\{\sin \left|\Delta \varphi_{\mathbf{h}}\right| \times\right. \\
& {\left.\left[\sum_{\mathbf{h}^{\prime}} m_{\mathbf{h}^{\prime}} m_{\mathbf{h}-\mathbf{h}^{\prime}} \kappa_{\mathbf{h}, \mathbf{h}^{\prime}} \sin \left(\Phi_{3}^{\prime}+\Delta \varphi_{\mathbf{h}^{\prime} \text { best }}+\Delta \varphi_{\mathbf{h}-\mathbf{h}^{\prime} \text { best }}\right)+x \sin \delta_{\mathbf{h}}\right]\right\} }
\end{aligned}
$$

where

$$
\begin{gathered}
\delta_{\mathbf{h}}=\varphi_{\mathbf{h}, p}-\varphi_{\mathbf{h}}^{\prime} \quad, \Delta \varphi_{\mathbf{h} \text { best }}=\varphi_{\mathbf{h} \text { best }}-\varphi_{\mathbf{h}}^{\prime}, \Phi_{3}^{\prime}=-\varphi_{\mathbf{h}}^{\prime}+\varphi_{\mathbf{h}^{\prime}}^{\prime}+\varphi_{\mathbf{h}^{\prime} \mathbf{h}^{\prime}}^{\prime} \\
\tan \left(\Delta \varphi_{\mathbf{h} \text { best }}\right)=2\left(P_{+}-\frac{1}{2}\right) \sin \left|\Delta \varphi_{\mathbf{h}}\right| / \cos \Delta \varphi_{\mathbf{h}} \\
m_{\mathbf{h}}=\exp \left(-\sigma_{\mathbf{h}}^{2} / 2\right)\left\{\left[2\left(P_{+}-\frac{1}{2}\right)^{2}+\frac{1}{2}\right]\left(1-\cos 2 \Delta \varphi_{\mathbf{h}}\right)+\cos 2 \Delta \varphi_{\mathbf{h}}\right\}^{1 / 2}
\end{gathered}
$$


The figure of merit $\mathrm{m}_{\mathbf{h}}$ is a measure of reliability of $\Delta \varphi_{\mathbf{h b e s t}} . \mathrm{m}_{\mathbf{h}}$ includes three factors: (i) $\exp \left(-\sigma_{\mathbf{h}}^{2} / 2\right)$, a measure of the sharpness of the phase-doublet distribution; (ii) $\left(P_{+}-1 / 2\right)^{2}$, a measure of the bias of $\Delta \varphi_{\mathbf{h}}$ towards positive or negative. It reaches the maximum value when $P_{+}$equals 0 or 1 ; (iii) $\cos 2 \Delta \varphi_{\mathrm{h}}$, a measure of the closeness of the two possible phases, $\varphi_{\mathrm{h}}+\Delta \varphi_{\mathrm{h}}$ and $\varphi_{\mathrm{h}}-\Delta \varphi_{\mathrm{h}}$. It reaches the maximum value when $\Delta \varphi_{\mathrm{h}}$ equals 0 or $\pi$. Either of the last two factors will have no effect on $m_{h}$ when the other one reaches the maximum value. A procedure for breaking the SIR or OAS phase ambiguity making use of equations (29), (30) and (31) has been proposed and is implemented in the program OASIS [27].

\section{Applications}

After a series of test calculations with error-free data, our method was first successfully applied to the OAS data of the protein aPP [14]. The first application to experimental SIR data was done with the protein Rhe [28]. The method was further combined with the solvent-flattening technique showing substantial improvement in dealing with experimental protein OAS and SIR data $[15,18]$. More recent results will be discussed in another lecture [29].

\section{References}

1. Coulter, C. L. (1965) A possible new method for the determination of crystallographic phases for proteins, J. Mol. Biol. 12, 292-295.

2. Fan, H. F. (1965) The use of sign relationship in the determination of heavy-atom-containing crystal structures I. A sign refinement procedure, Acta Phys. Sin. 21, 1105-1114 (in Chinese). (An English translation can be found in Chinese Physics (1965) 1418-1428.)

3. Fan, H. F. (1965) The use of sign relationship in the determination of heavy-atom-containing crystal structures II. Component relationship and its applications, Acta Phys. Sin. 21, 1114-1118 (in Chinese). (An English translation can be found in Chinese Physics (1965) 1429-1435.)

4. Karle, J. (1966) Isomorphous substitution and formulas for phase determination, Acta Cryst. 21, 273276.

5. Hendrickson, W. A. (1971) Some aids for breaking the phase ambiguity in the single isomorphous replacement method, Acta Cryst. B27, 1474-1475.

6. Hauptman, H. A. (1982) On integrating the technique of direct methods and isomorphous replacement I. The theoretical basis, Acta Cryst. A38, 289-294.

7. Hauptman, H. A., Potter, S. and Weeks, C. M. (1982) On integrating the technique of direct methods and isomorphous replacement II. The first applications, Acta Cryst. A38, 294-300.

8. Giacovazzo, C. (1983) The estimation of two-phase and three-phase invariants in $P 1$ when anomalous scatterers are present, Acta Cryst. A39, 585-592.

9. Fan, H. F. and Gu, Y. X. (1985) Combining Direct Methods with Isomorphous Replacement or Anomalous Scattering Data. III. The Incorporation of Partial Structure Information, Acta Cryst. A41, 280-284.

10. Fortier, S., Moore, N. J. and Fraser, M. E. (1985) A direct-methods solution to the phase problem in the single isomorphous replacement case: theoretical basis and initial applications, Acta Cryst. A41, 571577.

11. Klop, E. A., Krabbendam, H. and Kroon, J. (1987) On the direct-methods theory of single isomorphous replacement, Acta Cryst. A43, 810-820. 
12. Giacovazzo, C., Cascarano, G. and Zheng, C. D. (1988) On integrating the techniques of direct methods and isomorphous replacement. A new probabilistic formula for triplet invariants, Acta Cryst. A44, 4551.

13. Kyriakidis, C. E., Peschar, R. and Schenk, H. (1993) On the direct methods phase information from differences between isomorphous structure factors, Acta Cryst. A49, 557-569.

14. Fan, H.F., Hao, Q., Gu, Y.X., Qian, J.Z., Zheng, C.D. and Ke, H. (1990) Combining Direct Methods with Isomorphous Replacement or Anomalous Scattering Data. VII. Ab Initio Phasing of OneWavelength Anomalous Scattering Data from a Small Protein, Acta Cryst. A46, 935-939.

15. Sha, B.D., Liu S.P., Gu Y.X., Fan H.F., Ke H., Yao J.X. and M.M. Woolfson (1995) Direct Phasing of One-wavelength Anomalous-Scattering Data of the Protein Core Streptavidin, Acta Cryst. D51, 342346.

16. Giacovazzo, C., Siliqi, D. and Zanotti, G. (1995) The ab initio crystal structure solution of proteins by direct methods. III. The phase extension process, Acta Cryst. A51, 177-188.

17. Langs, D. A., Guo, D. and Hauptman, H. A. (1995) TDSIR phasing: direct use of phase-invariant distributions in macromolecular crystallography, Acta Cryst. A51, 535-542.

18. Zheng, X. F., Zheng, C. D., Gu, Y. X., Mo, Y. D., Fan, H.F. and Hao, Q. (1997) Use of single isomorphous replacement data of proteins - resolving the phase ambiguity and a new procedure for phase extension, Acta Cryst. D53, 49-55.

19. Fan, H. F., Han, F. S., Oian, J. Z. and Yao, J. X. (1984) Combining direct methods with isomorphous replacement or anomalous scattering data I, Acta Cryst. A40, 489-495.

20. Sayre, D. (1952) The squaring method: a new method for phase determination, Acta Cryst. 5, 60-65.

21. Grant, D. F., Howells, R. G. and Rogers, D. (1957) A method for the systematic application of sign relations, Acta Cryst. 10, 489-497.

22. Wang, B. C. (1981) Protein structure determination by the single isomorphous replacement method with a phase selection and refinement process, Acta Cryst. A37, C-11.

23. Wang, B. C. (1985) Diffraction Methods for Biological Macromolecules, in H. Wyckoff, C.H.W. Hirs and S.N. Timasheff (ed.), Methods in Enzymology, Vol. 115, Academic Press, New York, pp. 90-112.

24. Cochran, W. (1955) Relations between the phase of structure factors, Acta Cryst. 8, 473-478.

25. Sim, G. A. (1959) The distribution of phase angles for structures containing heavy atoms II. A modification of the normal heavy-atom method for non-centrosymmetrical structures, Acta Cryst. 12, 813-815.

26. Fan, H. F., Han, F. S. and Oian, J. Z. (1984) Combining direct methods with isomorphous replacement or anomalous scattering data II. The treatment of errors, Acta Cryst. A40, 495-498.

27. Hao, Q., Gu, Y. X., Zheng, C. D. and Fan, H. F. (1996) OASIS: a computer program for breaking the phase ambiguity in OAS or SIR protein data, School of Applied Sciences, De Montfort University, Leicester LE1 9BH, England and Institute of Physics, Chinese Academy of Sciences, Beijing 100080, P. R. China.

28. Fan, H. F. \& Wang, B. C. (1988). Unpublished work, partial results of which are quoted in Woolfson, M. M. and Fan, H. F. (1995) Physical and Non-physical Methods of Solving Crystal Structures, Cambridge University Press, Cambridge, pp. 164-166.

29. Fan, H. F., Hao, Q., Harvey I., Hasnain, S. S., Liu, Y. D., Gu, Y. X., Zheng, C. D. and Ke, H. (1997) Applications of direct methods with single isomorphous replacement or one-wavelength anomalous scattering data, in these Proceedings. 